Pure and Applied Mathematics Quarterly

Volume 5, Number 3

(Special Issue: In honor of

Friedrich Hirzebruch, Part 2 of 2$)$

1139-1159, 2009

\title{
Rigidity Theorems on Odd Dimensional Manifolds
}

\author{
Kefeng Liu and Yong Wang \\ Dedicated to Professor F. Hirzebruch on the occasion of his 80th birthday
}

\begin{abstract}
We prove several rigidity theorems for elliptic genera associated to Toeplitz operators on odd dimensional spin manifolds. We also prove an equivariant odd index theorem for Dirac operators with involution parity and the Atiyah-Hirzebruch vanishing theorems for odd dimensional spin manifolds.
\end{abstract}

Keywords: Toeplitz operators; Equivariant odd index theorem; Rigidity theorem; Involution

\section{INTRODUCTION}

One of the most interesting applications of the Atiyah-Bott-Segal-Singer fixed point formula is the vanishing theorem of Atiyah and Hirzebruch [AH]. This theorem was vastly generalized to vanishing and rigidity theorems for elliptic genera, see [BT], [Li1], [Li2] and [LiM]. The purpose of this paper is to prove similar results for odd dimensional manifolds. We will first derive fixed point formulas on odd dimensional manifolds, then we combine them with the modularity arguments to prove vanishing and rigidity theorems for elliptic genera associated to Toeplitz operators on odd dimensional manifolds. We believe our results should have applications to the study of topology of odd dimensional manifolds.

Received September 20, 2007. 
In Section 2.1, we state an equivariant odd index theorem for Toeplitz operators and prove an Atiyah-Hirzebruch type theorem for odd dimensional manifolds. Rigidity theorems for ellipitc genera on odd dimensional manifolds stated for Toeplitz operators are given in Section 2.2. In Section 3, the rigidity theorems are generalized to the nonzero anomaly case. In Section 4 , we prove an equivariant odd index theorem for Dirac operators with involution parity which is a generalization of Freed's odd index theorem in $[\mathrm{Fr}]$ and another Atiyah-Hirzebruch type theorem for odd dimensional manifolds is given.

\section{Rigidity THEOREMS FOR TOEPLITZ OPERATORS ON ODD DIMENSIONAL MANIFOLDS}

\subsection{An Atiyah-Hirzebruch vanishing theorem for odd dimensional man- ifolds}

In this section, we first state a generalization of the equivariant odd index theorem for Toeplitz operators proved by Fang in [Fa] (for more details, see [Fa]). Let $X$ be a closed oriented spin manifold of dimension $2 r+1$, with a fixed spin structure. Let $\triangle(T X)$ be the canonical complex spinor bundle of $X$ and $E$ be a complex Hermitian vector bundle with a Hermitian connection $\nabla^{E}$. Let $D^{E}$ be the associated twisted Dirac operator which is a self adjoint first order elliptic differential operator acting on $\Gamma(\triangle(T X) \otimes E$ ) (the smooth sections space of $\triangle(T X) \otimes E)$, so it induces a spectral decomposition of $L^{2}(\triangle(T X) \otimes E)$ which is the $L^{2}$ completion of $\Gamma(\triangle(T X) \otimes E)$. Denote by $L_{+}^{2}(\triangle(T X) \otimes E)$ the direct sum of eigenspaces of $D^{E}$ associated to nonnegative eigenvalues, and by $P^{+}$the orthogonal projection operator from $L^{2}(\triangle(T X) \otimes E)$ to $L_{+}^{2}(\triangle(T X) \otimes E)$. Given a trivial complex vector bundle $\mathbf{C}^{N}$ over $X, D^{E}$ and $P_{+}$extend trivially as operators on $\Gamma\left(\triangle(T X) \otimes E \otimes \mathbf{C}^{N}\right)$. Let $g: X \rightarrow U(N)$ be a smooth map. Then $g$ extends to an action on $\Gamma\left(\triangle(T X) \otimes E \otimes C^{N}\right)$ as $\operatorname{Id}_{(\triangle(T X) \otimes E)} \otimes g$, still denoted by $g$.

Definition 2.1. The Toeplitz operator associated to $D^{E}$ and $g$ is

$T_{g} \otimes E=\left(P_{+} \otimes I d_{\mathbf{C}^{N}}\right) g\left(P_{+} \otimes I d_{\mathbf{C}^{N}}\right): L_{+}^{2}\left(\triangle(T X) \otimes E \otimes \mathbf{C}^{N}\right) \rightarrow L_{+}^{2}\left(\triangle(T X) \otimes E \otimes \mathbf{C}^{N}\right)$.

It is a classical fact that $T_{g} \otimes E$ is a bounded Fredholm operator between the given Hilbert spaces. Next, we will state the equivariant index theorem for Toeplitz 
operators.

Consider a compact group $\widetilde{H}$ of isometries of $X$ preserving the orientation and spin structure. Let $E$ be a $\widetilde{H}$-vector bundle with $\widetilde{H}$-invariant Hermitian connection. There is a lift of $h \in \widetilde{H}$ acting on $\Gamma(\triangle(T X) \otimes E)$ which commutes with $D^{E}$, so it commutes with $P_{+}$. We also assume that

$$
g(h x)=g(x), \text { for any } h \in \widetilde{H} \text { and any } x \in X,
$$

Then,

$$
\left(T_{g} \otimes E\right) h=h\left(T_{g} \otimes E\right) .
$$

Definition 2.2. The equivariant index associated to $T_{g} \otimes E$ and $h$ is

$$
\operatorname{Ind}\left(h, T_{g} \otimes E\right)=\operatorname{tr}\left(h \mid \operatorname{ker}\left(T_{g} \otimes E\right)\right)-\operatorname{tr}\left(h \mid \operatorname{coker}\left(T_{g} \otimes E\right)\right) .
$$

Let $F_{\alpha}$ be the fixed point submanifolds of $X$ under the action of any $h \in \widetilde{H}$ and for simplicity, we assume only one fixed point component $F$ (the same discussions for many components), and $N(F)$ be the normal bundle of $F$ in $T X$. Let $\operatorname{dim} F=2 q_{0}+1$ and $\operatorname{dim} N(F)=2 s$. In any local coordinate system, $R^{N(F)}$ is the curvature matrix of the bundle $N(F)$ and $\Theta$ is the rotation matix of $h$ acting on $N(F)$. Let $\hat{A}(F)$ and $\operatorname{ch}_{h}(E)$ be the $\hat{A}$ characteristic form on $T F$ and the equivariant Chern character of $E$ respectively. Let $\operatorname{ch}(g)$ be the odd Chern character of $g$. The following theorem is a generalization of Fang' odd equivariant index theorem, its proof follows the same argument as in [Fa].

Theorem 2.3. We have

$\operatorname{Ind}\left(h, T_{g} \otimes E\right)=\left(\left(\frac{-\sqrt{-1}}{2 \pi}\right)^{q_{0}+1} \operatorname{ch}(g) \widehat{A}(F)\left[\operatorname{Pf}\left(2 \sin \left(\frac{R^{N(F)}}{4 \pi \sqrt{-1}}+\frac{1}{2} \Theta\right)\right)\right]^{-1} \operatorname{ch}_{h}(E)\right)[F]$

Next we assume $\widetilde{H}=S^{1}$ and let $h=e^{2 \pi i t}$ be a generator of the circle group. Then the tangent bundle $T X$ and $E$ have decompositions into sum :

$$
\left.T X\right|_{F}=E_{1} \oplus \cdots \oplus E_{s} \oplus T F ;\left.E\right|_{F}=L_{1} \oplus \cdots \oplus L_{l_{0}},
$$

where $E_{1} \cdots E_{s}$ are $S^{1}$-invariant 2-planes and $L_{1} \cdots L_{l_{0}}$ are complex line bundles. Assume that $h$ acts on $E_{j}$ and $L_{l}$ by $e^{2 \pi i t m_{j}}$ and $e^{2 \pi i t c_{l}}$ respectively. Let $2 \pi \sqrt{-1} y_{l},-2 \pi \sqrt{-1} y_{l},\left(1 \leq l \leq q_{0}\right)$ be the Chern roots of $T F \otimes C$ and 
$2 \pi \sqrt{-1} x_{j},-2 \pi \sqrt{-1} x_{j},(1 \leq l \leq s)$ be the Chern roots of $N F \otimes C$ and $2 \pi \sqrt{-1} z_{\nu},(1 \leq$ $\nu \leq l_{0}$ ) be the Chern roots of $\left.E\right|_{F}$. Now we give the Chern root expression of odd equivariant index theorem for $\widetilde{H}=S^{1}$ case.

$$
\begin{gathered}
\operatorname{Ind}\left(h, T_{g} \otimes E\right)=\left(\left(\frac{-\sqrt{-1}}{2 \pi}\right)^{q_{0}+1} \frac{1}{(-2)^{s}} \operatorname{ch}(g) \prod_{l=1}^{q_{0}} \frac{\pi y_{l}}{\sin \pi y_{l}}\right. \\
\left.\quad \times \prod_{j=1}^{s} \frac{1}{\sin \left[\pi\left(x_{j}+m_{j} t\right)\right]} \sum_{\nu=1}^{l_{0}} e^{2 \pi \sqrt{-1}\left(c_{\nu} t+z_{\nu}\right)}\right)[F]
\end{gathered}
$$

Using (2.5), by the same method as in $[\mathrm{AH}]$ or $[\mathrm{BT}]$, we can prove an AtiyahHirzebruch type theorem for odd dimensional spin manifolds:

Theorem 2.4. Let $X$ be an odd dimensional connected spin manifold which admits an nontrivial $S^{1}$ action and $g: X \rightarrow U(N)$ satisfying $g(h x)=g(x)$, for any $h \in$ $S^{1}$ and any $x \in X$, then we have

$$
\int_{X} \widehat{A}(T X) \operatorname{ch}(g)=0
$$

Proof. Let $h=e^{2 \pi i t}$ be the topological generator of $S^{1}$ where $t$ is a irrational number. Let $\Gamma$ be the subgroup of $S^{1}$ generated by $h$, then the closure of $\Gamma$ is $S^{1}$ and fixed point sets $F(h)=F\left(h^{m}\right)=F\left(S^{1}\right)$ for any $m \in \mathbf{N}$. Since $\operatorname{ind}_{S^{1}}\left(T_{g}\right):=\left[\operatorname{ker} T_{g}\right]-\left[\operatorname{coker} T_{g}\right]$ is a virtual representative space of $S^{1}$, then its character

$$
\operatorname{ind}_{z_{0}}\left(T_{g}\right)=\sum_{k=-N}^{N} n_{k} z_{0}^{k}
$$

where $z_{0} \in S^{1}$ and $k, n_{k}$ are integers. By (2.4) and (2.5) for untwisted case, for any $z=h^{m} \in \Gamma$, we have up to a constant

$$
\operatorname{ind}_{z}\left(T_{g}\right)=\int_{F} \operatorname{ch}(g) \widehat{A}\left(F\left(S^{1}\right)\right) \sum_{j=1}^{s} \frac{1}{z^{m_{j} / 2} e^{i \pi x_{j}}-z^{-m_{j} / 2} e^{-i \pi x_{j}}} .
$$

Considering the Taylor expansion of $\frac{1}{z^{m_{j} / 2} e^{x}-z^{-m_{j} / 2} e^{-x}}$, the right hand of (2.8) equals

$$
\sum_{j=1}^{s} \frac{1}{z^{m_{j} / 2}-z^{-m_{j} / 2}} \times f\left(\frac{z^{m_{j} / 2}+z^{-m_{j} / 2}}{z^{m_{j} / 2}-z^{-m_{j} / 2}}\right)
$$


where $f\left(\frac{z^{m_{j} / 2}+z^{-m_{j} / 2}}{z^{m_{j} / 2}-z^{-m_{j} / 2}}\right)$ is a polynomial on $\frac{z^{m_{j} / 2}+z^{-m_{j} / 2}}{z^{m_{j} / 2}-z^{-m_{j} / 2}}$. We extend the right hand of (2.7) and (2.9) as meromorphic functions on the complex plane. Since they are equal on $\Gamma$, they must be equal on the complex plane. $\sum_{k=-N}^{N} n_{k} z^{k}$ has poles at $0, \infty$ and (2.9) has poles at $|z|=1$, so they have no poles on $\mathbf{C} \cup \infty$ and are constant. When $z$ goes to zero, then (2.9) is zero. So $\operatorname{ind}_{z_{0}}\left(T_{g}\right)$ is zero, especially,

$$
\operatorname{ind}\left(T_{g}\right)=\int_{X} \widehat{A}(T X) \operatorname{ch}(g)=0 .
$$

\subsection{Rigidity theorems of elliptic genera for Toeplitz operators}

First we recall the Witten elements. For a vector bundle $E$ on $X$, let

$$
\begin{aligned}
& S_{t}(E)=1+t E+t^{2} S^{2} E+\cdots, \\
& \wedge_{t}(E)=1+t E+t^{2} \wedge^{2} E+\cdots
\end{aligned}
$$

be the symmetric and exterior power operations in $K(M)[t]$. Let

$$
\begin{gathered}
\Theta_{q}^{\prime}(T X)=\otimes_{n=1}^{\infty} \wedge_{q^{n}}(T X) \otimes_{m=1}^{\infty} S_{q^{m}}(T X), \\
\Theta_{q}(T X)=\otimes_{n=1}^{\infty} \wedge_{-q^{n-\frac{1}{2}}}(T X) \otimes_{m=1}^{\infty} S_{q^{m}}(T X), \\
\Theta_{-q}(T X)=\otimes_{n=1}^{\infty} \wedge_{q^{n-\frac{1}{2}}}(T X) \otimes_{m=1}^{\infty} S_{q^{m}}(T X) .
\end{gathered}
$$

Furthermore, let $V$ be a real vector bundle on $X$ with structure group $\operatorname{Spin}(2 k)$ and the action lift to $V$. So we have

$$
\left.V\right|_{F}=V_{1} \oplus \cdots \oplus V_{k}
$$

where $V_{1} \cdots V_{k}$ are $S^{1}$-invariant 2-planes and $h$ acts on $V_{\nu}$ by $e^{2 \pi i t n_{\nu}}$. Denote the Chern root of $V_{\nu}$ by $2 \pi i u_{\nu}$. Let

$$
\begin{gathered}
\Theta_{q}^{\prime}(T X \mid V)=\otimes_{n=1}^{\infty} \wedge_{q^{n}}(V) \otimes_{m=1}^{\infty} S_{q^{m}}(T X), \\
\Theta_{q}(T X \mid V)=\otimes_{n=1}^{\infty} \wedge_{-q^{n-\frac{1}{2}}}(V) \otimes_{m=1}^{\infty} S_{q^{m}}(T X), \\
\left.\Theta_{-q}(T X \mid V)=\otimes_{n=1}^{\infty} \wedge_{q^{n-\frac{1}{2}}}(V) \otimes_{m=1}^{\infty} S_{q^{m}}(T X)\right), \\
\Theta_{q}^{\star}(T X \mid V)=\otimes_{n=1}^{\infty} \wedge_{-q^{n}}(V) \otimes_{m=1}^{\infty} S_{q^{m}}(T X) .
\end{gathered}
$$

Let $p_{1}(.)_{S^{1}}$ denote the first $S^{1}$-equivariant Pontrjagin class and $\Delta(V)=\Delta^{-}(V) \oplus$ $\Delta^{+}(V)$ be the spinor bundle of $V$, then we have the following rigidity theorems. 
Theorem 2.5. a) For an odd dimensional connected spin manifold with nontrivial $S^{1}$ action, assume that it has only 1-dimensional fixed submanifolds, then the Toeplitz operators $T_{g} \otimes \Delta(T X) \otimes \Theta_{q}^{\prime}(T X), T_{g} \otimes \Theta_{q}(T X), T_{g} \otimes \Theta_{-q}(T X)$ are rigid.

b) If the action lifts to $V$ and $p_{1}(V)_{S^{1}}=p_{1}(X)_{S^{1}}$, the operators $T_{g} \otimes \Delta(V) \otimes$ $\Theta_{q}^{\prime}(T X \mid V), T_{g} \otimes\left(\Delta^{+}(V)-\Delta^{-}(V)\right) \otimes \Theta_{q}^{\star}(T X \mid V), T_{g} \otimes \Theta_{q}(T X \mid V), T_{g} \otimes \Theta_{-q}(T X \mid V)$ are rigid.

Example 1. Let $M$ be an even dimensional spin manifold with nontrivial $S^{1}$ action preserving orientation and spin structure and its fixed points set is isolated. Let $X=M \times S^{1}$ with $S^{1}$ acting on $X$ by trivial action on $S^{1}$. $g$ is a smooth function on $S^{1}$.

$$
\begin{aligned}
& \text { For } \tau \in \mathbf{H}=\{\tau \in \mathbf{C} ; \operatorname{Im} \tau>0\}, q=e^{2 \pi i \tau}, \text { let } \\
& \qquad \theta_{3}(v, \tau)=c(q) \prod_{n=1}^{\infty}\left(1+q^{n-\frac{1}{2}} e^{2 \pi i v}\right) \prod_{n=1}^{\infty}\left(1+q^{n-\frac{1}{2}} e^{-2 \pi i v}\right), \\
& \theta_{2}(v, \tau)=c(q) \prod_{n=1}^{\infty}\left(1-q^{n-\frac{1}{2}} e^{2 \pi i v}\right) \prod_{n=1}^{\infty}\left(1-q^{n-\frac{1}{2}} e^{-2 \pi i v}\right), \\
& \theta_{1}(v, \tau)=c(q) q^{\frac{1}{8}} 2 \cos (\pi v) \prod_{n=1}^{\infty}\left(1+q^{n} e^{2 \pi i v}\right) \prod_{n=1}^{\infty}\left(1+q^{n} e^{-2 \pi i v}\right), \\
& \theta(v, \tau)=c(q) q^{\frac{1}{8}} 2 \sin (\pi v) \prod_{n=1}^{\infty}\left(1-q^{n} e^{2 \pi i v}\right) \prod_{n=1}^{\infty}\left(1-q^{n} e^{-2 \pi i v}\right),
\end{aligned}
$$

be the classical theta functions (see $[\mathrm{Ch}]$ ), where $c(q)=\prod_{n=1}^{\infty}\left(1-q^{n}\right)$.

Now we define some functions on $\mathbf{C} \times \mathbf{H}$

$$
\begin{aligned}
& F_{d_{s}}(t, \tau)=\operatorname{ch}(g) \prod_{l=1}^{q_{0}} \frac{\pi y_{l} \theta_{1}\left(y_{l}, \tau\right)}{\theta\left(y_{l}, \tau\right)} \prod_{j=1}^{s} \frac{\theta_{1}\left(m_{j} t+x_{j}, \tau\right)}{\theta\left(m_{j} t+x_{j}, \tau\right)}[F], \\
& F_{D}(t, \tau)=\operatorname{ch}(g) \prod_{l=1}^{q_{0}} \frac{\pi y_{l} \theta_{2}\left(y_{l}, \tau\right)}{\theta\left(y_{l}, \tau\right)} \prod_{j=1}^{s} \frac{\theta_{2}\left(m_{j} t+x_{j}, \tau\right)}{\theta\left(m_{j} t+x_{j}, \tau\right)}[F], \\
& F_{-D}(t, \tau)=\operatorname{ch}(g) \prod_{l=1}^{q_{0}} \frac{\pi y_{l} \theta_{3}\left(y_{l}, \tau\right)}{\theta\left(y_{l}, \tau\right)} \prod_{j=1}^{s} \frac{\theta_{3}\left(m_{j} t+x_{j}, \tau\right)}{\theta\left(m_{j} t+x_{j}, \tau\right)}[F],
\end{aligned}
$$




$$
\begin{aligned}
& F_{d_{s}}^{V}(t, \tau)=\operatorname{ch}(g) \prod_{l=1}^{q_{0}} \frac{\pi y_{l}}{\theta\left(y_{l}, \tau\right)} \frac{\prod_{\nu=1}^{k} \theta_{1}\left(n_{\nu} t+u_{\nu}, \tau\right)}{\prod_{j=1}^{s} \theta\left(m_{j} t+x_{j}, \tau\right)}[F], \\
& F_{D}^{V}(t, \tau)=\operatorname{ch}(g) \prod_{l=1}^{q_{0}} \frac{\pi y_{l}}{\theta\left(y_{l}, \tau\right)} \frac{\prod_{\nu=1}^{k} \theta_{2}\left(n_{\nu} t+u_{\nu}, \tau\right)}{\prod_{j=1}^{s} \theta\left(m_{j} t+x_{j}, \tau\right)}[F], \\
& F_{-D}^{V}(t, \tau)=\operatorname{ch}(g) \prod_{l=1}^{q_{0}} \frac{\pi y_{l}}{\theta\left(y_{l}, \tau\right)} \frac{\prod_{\nu=1}^{k} \theta_{2}\left(n_{\nu} t+u_{\nu}, \tau\right)}{\prod_{j=1}^{s} \theta\left(m_{j} t+x_{j}, \tau\right)}[F], \\
& F_{D^{\star}}^{V}(t, \tau)=\operatorname{ch}(g) \prod_{l=1}^{q_{0}} \frac{\pi y_{l}}{\theta\left(y_{l}, \tau\right)} \frac{\prod_{\nu=1}^{k} \theta\left(n_{\nu} t+u_{\nu}, \tau\right)}{\prod_{j=1}^{s} \theta\left(m_{j} t+x_{j}, \tau\right)}[F] .
\end{aligned}
$$

By (2.5) and [LaM,page 238], for any irrational number $t \in[0,1]$ and $h=e^{2 \pi i t}$, up to some constants, we get

$$
\begin{gathered}
\operatorname{Ind}_{h}\left(T_{g} \otimes \Delta(T X) \otimes \Theta_{q}^{\prime}(T X)\right)=F_{d_{s}}(t, \tau), \\
\operatorname{Ind}_{h}\left(T_{g} \otimes \Theta_{q}(T X)\right)=q^{\frac{r}{8}} F_{D}(t, \tau), \\
\operatorname{Ind}_{h}\left(T_{g} \otimes \Theta_{-q}(T X)\right)=q^{\frac{r}{8}} F_{-D}(t, \tau), \\
\operatorname{Ind}_{h}\left(T_{g} \otimes \Delta(V) \otimes \Theta_{q}^{\prime}(T X \mid V)\right)=\left(c(q) q^{\frac{1}{8}}\right)^{r-k} F_{d_{s}}^{V}(t, \tau), \\
\operatorname{Ind}_{h}\left(T_{g} \otimes \Theta_{q}(T X \mid V)\right)=q^{\frac{r}{8}}(c(q))^{r-k} F_{D}^{V}(t, \tau), \\
\operatorname{Ind}_{h}\left(T_{g} \otimes \Theta_{-q}(T X \mid V)\right)=q^{\frac{r}{8}}(c(q))^{r-k} F_{-D}^{V}(t, \tau), \\
\operatorname{Ind}_{h}\left(T_{g} \otimes\left(\Delta^{+}(V)-\Delta^{-}(V)\right) \otimes \Theta_{q}^{\star}(T X \mid V)\right)=\left(c(q) q^{\frac{1}{8}}\right)^{r-k} F_{D^{\star}}^{V}(t, \tau)
\end{gathered}
$$

As in [Li1], [LiM], we extend these $F$ and $\left(F^{V}\right)$ to meromorphic functions on $\mathbf{C} \times \mathbf{H}$, then the rigidity theorems are equivariant to the statement that these $F$ and $F^{V}$ are holomorphic by the following lemma

Lemma 2.6. a) $F_{d_{s}}(t, \tau), F_{D}(t, \tau)$ and $F_{-D}(t, \tau)$ are invariant under the action $U: t \rightarrow t+a \tau+b$. for $a, b \in 2 \mathbf{Z}$

b) If $p_{1}(V)_{S^{1}}=p_{1}(X)_{S^{1}}$, then $F_{d_{s}}^{V}(t, \tau), F_{D}^{V}(t, \tau), F_{-D}^{V}(t, \tau)$ and $F_{D^{\star}}^{V}(t, \tau)$ are invariant under $U$.

Proof. Recall that we have the following transformation formulas of thetafunctions (see $[\mathrm{Ch}])$ :

$$
\begin{aligned}
\theta(t+1, \tau) & =-\theta(t, \tau) ; \theta(t+\tau, \tau)=-q^{-\frac{1}{2}} e^{-2 \pi i t} \theta(t, \tau), \\
\theta_{1}(t+1, \tau) & =-\theta_{1}(t, \tau) ; \theta_{1}(t+\tau, \tau)=q^{-\frac{1}{2}} e^{-2 \pi i t} \theta_{1}(t, \tau), \\
\theta_{2}(t+1, \tau) & =\theta_{2}(t, \tau) ; \theta_{2}(t+\tau, \tau)=-q^{-\frac{1}{2}} e^{-2 \pi i t} \theta_{2}(t, \tau),
\end{aligned}
$$




$$
\theta_{3}(t+1, \tau)=\theta_{3}(t, \tau) ; \theta_{3}(t+\tau, \tau)=q^{-\frac{1}{2}} e^{-2 \pi i t} \theta_{3}(t, \tau),
$$

From these formulas, for $\theta_{\nu}=\theta, \theta_{1}, \theta_{2}, \theta_{3}$ and $(a, b) \in(2 \mathbf{Z})^{2}, l \in \mathbf{Z}$, we get

$$
\theta_{\nu}(x+l(t+a \tau+b), \tau)=e^{-\pi i\left(2 l a x+2 l^{2} a t+l^{2} a^{2} \tau\right)} \theta_{\nu}(x+l t, \tau),
$$

which proves (a).

To prove (b), note that since $p_{1}(V)_{S^{1}}=p_{1}(T X)_{S^{1}}$, we have

$$
\sum_{\nu=1}^{k}\left(u_{\nu}+n_{\nu} t\right)^{2}=\sum_{l=1}^{q_{0}} y_{l}^{2}+\sum_{j=1}^{s}\left(x_{j}+m_{j} t\right)^{2} .
$$

This implies the equalities:

$$
\sum_{\nu=1}^{k} n_{\nu} u_{\nu}=\sum_{j=1}^{s} m_{j} x_{j} ; \sum_{\nu=1}^{k} n_{\nu}^{2}=\sum_{j=1}^{s} m_{j}^{2} .
$$

By $(2.16),(2.19)$ and $(2.21)$, we can prove b).

Let $\Phi_{\tau}$ be scaling homomorphism from $\wedge\left(T^{\star} F\right)$ into itself: $\beta^{[m]} \rightarrow \tau^{\frac{1-m}{2}} \beta^{[m]}$. Write

$$
\begin{aligned}
& F_{d_{s}}(t, \tau)^{\left[m_{0}\right]}=\operatorname{ch}(g)^{\left[m_{0}\right]} \prod_{l=1}^{q_{0}} \frac{\pi y_{l} \theta_{1}\left(y_{l}, \tau\right)}{\theta\left(y_{l}, \tau\right)} \prod_{j=1}^{s} \frac{\theta_{1}\left(m_{j} t+x_{j}, \tau\right)}{\theta\left(m_{j} t+x_{j}, \tau\right)}[F], \\
& \Phi_{\tau}^{\star} F_{d_{s}}(t, \tau)=\Phi_{\tau}[\operatorname{ch}(g)] \prod_{l=1}^{q_{0}} \frac{\pi y_{l} \theta_{1}\left(y_{l}, \tau\right)}{\theta\left(y_{l}, \tau\right)} \prod_{j=1}^{s} \frac{\theta_{1}\left(m_{j} t+x_{j}, \tau\right)}{\theta\left(m_{j} t+x_{j}, \tau\right)}[F],
\end{aligned}
$$

where $\operatorname{ch}(g)^{\left[m_{0}\right]}$ denotes the $\left[m_{0}\right]$-degree component of $\operatorname{ch}(g)$. Similarly we can define $F_{D}(t, \tau)^{\left[m_{0}\right]}, F_{-D}(t, \tau)^{\left[m_{0}\right]}$ and $\Phi_{\tau}^{\star} F_{D}(t, \tau), \Phi_{\tau}^{\star} F_{-D}(t, \tau)$,

For $g_{0}=\left(\begin{array}{ll}a & b \\ c & d\end{array}\right) \in S L_{2}(Z)$, we define its modular transformation on $\mathbf{C} \times \mathbf{H}$ by

$$
g_{0}(t, \tau)=\left(\frac{t}{c \tau+d}, \frac{a \tau+b}{c \tau+d}\right) .
$$

The two generators of $S L_{2}(Z)$ are

$$
S=\left(\begin{array}{cc}
0 & -1 \\
1 & 0
\end{array}\right), T=\left(\begin{array}{ll}
1 & 1 \\
0 & 1
\end{array}\right),
$$

which act on $\mathbf{C} \times \mathbf{H}$ in the following way:

$$
S(t, \tau)=\left(\frac{t}{\tau},-\frac{1}{\tau}\right), T(t, \tau)=(t, \tau+1) .
$$


Lemma 2.7. a) We have following identities:

$$
\begin{gathered}
F_{d_{s}}\left(\frac{t}{\tau},-\frac{1}{\tau}\right)=i^{r} \Phi_{\tau}^{\star} F_{D}(t, \tau) ; F_{d_{s}}(t, \tau+1)=F_{d_{s}}(t, \tau), \\
F_{-D}\left(\frac{t}{\tau},-\frac{1}{\tau}\right)=i^{r} \Phi_{\tau}^{\star} F_{-D}(t, \tau) ; F_{D}(t, \tau+1)=F_{-D}(t, \tau) e^{-\left(\frac{\pi i}{4}\right) r} .
\end{gathered}
$$

b) If $p_{1}(V)_{S^{1}}=p_{1}(X)_{S^{1}}$, then

$$
\begin{gathered}
F_{d_{s}}^{V}\left(\frac{t}{\tau},-\frac{1}{\tau}\right)=\left(\frac{\tau}{i}\right)^{(k-r) / 2} i^{r} \Phi_{\tau}^{\star} F_{d_{s}}^{V}(t, \tau) ; F_{d_{s}}^{V}(t, \tau+1)=e^{-\left(\frac{\pi i}{4}\right)(r-k)} F_{d_{s}}^{V}(t, \tau), \\
F_{-D}^{V}\left(\frac{t}{\tau},-\frac{1}{\tau}\right)=\left(\frac{\tau}{i}\right)^{(k-r) / 2} i^{r} \Phi_{\tau}^{\star} F_{-D}^{V}(t, \tau) ; F_{D}^{V}(t, \tau+1)=e^{-\left(\frac{\pi i}{4}\right) r} F_{-D}^{V}(t, \tau), \\
F_{D^{\star}}^{V}\left(\frac{t}{\tau},-\frac{1}{\tau}\right)=\left(\frac{\tau}{i}\right)^{(k-r) / 2} i^{r-k} \Phi_{\tau}^{\star} F_{D^{\star}}^{V}(t, \tau) ; F_{D^{\star}}^{V}(t, \tau+1)=e^{-\left(\frac{\pi i}{4}\right)(r-k)} F_{D^{\star}}^{V}(t, \tau) .
\end{gathered}
$$

Proof: By $[\mathrm{Ch}]$, we have the following transformation formula for the Jacobi theta-functions:

$$
\begin{gathered}
\theta\left(\frac{t}{\tau},-\frac{1}{\tau}\right)=\frac{1}{i} \sqrt{\frac{\tau}{i}} e^{\pi i t^{2} / \tau} \theta(t, \tau) ; \theta(t, \tau+1)=e^{\pi i / 4} \theta(t, \tau), \\
\theta_{1}\left(\frac{t}{\tau},-\frac{1}{\tau}\right)=\sqrt{\frac{\tau}{i}} e^{\pi i t^{2} / \tau} \theta_{2}(t, \tau) ; \theta_{1}(t, \tau+1)=e^{\pi i / 4} \theta_{1}(t, \tau) \\
\theta_{2}\left(\frac{t}{\tau},-\frac{1}{\tau}\right)=\sqrt{\frac{\tau}{i}} e^{\pi i t^{2} / \tau} \theta_{1}(t, \tau) ; \theta_{2}(t, \tau+1)=\theta_{3}(t, \tau), \\
\theta_{3}\left(\frac{t}{\tau},-\frac{1}{\tau}\right)=\sqrt{\frac{\tau}{i}} e^{\pi i t^{2} / \tau} \theta_{3}(t, \tau) ; \theta_{3}(t, \tau+1)=\theta_{2}(t, \tau)
\end{gathered}
$$


The action of $T$ on the functions on $F$ and $F^{V}$ is easy, we omit it. By (2.17), we have

$$
\begin{aligned}
& F_{d_{s}}\left(\frac{t}{\tau},-\frac{1}{\tau}\right) \\
&= \operatorname{ch}(g) \prod_{l=1}^{q_{0}} \frac{\pi y_{l} \theta_{1}\left(y_{l},-\frac{1}{\tau}\right)}{\theta\left(y_{l},-\frac{1}{\tau}\right)} \prod_{j=1}^{s} \frac{\theta_{1}\left(m_{j} t / \tau+x_{j},-\frac{1}{\tau}\right)}{\theta\left(m_{j} t / \tau+x_{j},-\frac{1}{\tau}\right)}[F] \\
&= i^{r} \operatorname{ch}(g) \prod_{l=1}^{q_{0}} \frac{\pi y_{l} \theta_{2}\left(\tau y_{l}, \tau\right)}{\theta\left(\tau y_{l}, \tau\right)} \prod_{j=1}^{s} \frac{\theta_{2}\left(m_{j} t+\tau x_{j}, \tau\right)}{\theta\left(m_{j} t+\tau x_{j}, \tau\right)}[F] \\
&= i^{r} \sum_{m_{0}=1}^{2 q_{0}+1} \operatorname{ch}(g)^{\left[m_{0}\right]}\left[\prod_{l=1}^{q_{0}} \frac{\pi y_{l} \theta_{2}\left(\tau y_{l}, \tau\right)}{\theta\left(\tau y_{l}, \tau\right)} \prod_{j=1}^{s} \frac{\theta_{2}\left(m_{j} t+\tau x_{j}, \tau\right)}{\theta\left(m_{j} t+\tau x_{j}, \tau\right)}\right]^{\left[2 q_{0}+1-m_{0}\right]} \\
&= i^{r} \sum_{m_{0}=1}^{2 q_{0}+1} \operatorname{ch}(g)^{\left[m_{0}\right]} \frac{1}{\tau^{q_{0}}}\left[\prod_{l=1}^{q_{0}} \frac{\pi \tau y_{l} \theta_{2}\left(\tau y_{l}, \tau\right)}{\theta\left(\tau y_{l}, \tau\right)} \prod_{j=1}^{s} \frac{\theta_{2}\left(m_{j} t+\tau x_{j}, \tau\right)}{\theta\left(m_{j} t+\tau x_{j}, \tau\right)}\right]^{\left[2 q_{0}+1-m_{0}\right]} \\
&= i^{r} \sum_{m_{0}=1}^{2 q_{0}+1} \operatorname{ch}(g)^{\left[m_{0}\right]} \frac{1}{\tau^{q_{0}}} \tau^{\frac{2 q_{0}+1-m_{0}}{2}}\left[\prod_{l=1}^{q_{0}} \frac{\pi y_{l} \theta_{2}\left(y_{l}, \tau\right)}{\theta\left(y_{l}, \tau\right)} \prod_{j=1}^{s} \frac{\theta_{2}\left(m_{j} t+x_{j}, \tau\right)}{\theta\left(m_{j} t+x_{j}, \tau\right)}\right]^{\left[2 q_{0}+1-m_{0}\right]} \\
&= i^{r} \sum_{m_{0}=1}^{2 q_{0}+1} \operatorname{ch}(g)^{\left[m_{0}\right]} \tau^{\frac{1-m_{0}}{2}} \prod_{l=1}^{q_{0}} \frac{\pi y_{l} \theta_{2}\left(y_{l}, \tau\right)}{\theta\left(y_{l}, \tau\right)} \prod_{j=1}^{s} \frac{\theta_{2}\left(m_{j} t+x_{j}, \tau\right)}{\theta\left(m_{j} t+x_{j}, \tau\right)}[F] \\
&= i^{r} \Phi_{\tau}^{*} F_{D}(t, \tau) .(2.30) \\
&
\end{aligned}
$$

Using the same trick, we can get other identities.

Remark. When $\operatorname{dim} F=1$, then $m_{0}=1$ and there is no $\Phi_{\tau}^{*}$ in Lemma 2.7.

Lemma 2.8 If $T X$ and $V$ are spin, then all of $F$ and $F^{V}$ above are holomorphic in $(t, \tau) \in \mathbf{R} \times \mathbf{H}$.

Proof. The proof is the same as the proof of Lemma 2.3 in [LiM] except that we use Theorem 2.3 instead of their equivariant family index theorem.

Proof of Theorem 2.5. We prove that these $F$ and $F^{V}$ are holomorphic on $\mathbf{C} \times \mathbf{H}$ which implies the rigidity of Theorem 2.5. We denote by $F$ one of the functions: 
$\left\{F, F^{V}\right\}$. Since $\operatorname{ch}(g)$ does not add poles, we know the possible polar divisors of $F$ in $\mathbf{C} \times \mathbf{H}$ are of the form $t=(n / l)(c \tau+d)$ with $n, c, d, l$ integers and $(c, d)=1$ or $c=1$ and $d=0$.

We can always find integers $a, b$ such that $a d-b c=1$. Then the matrix $g_{0}=\left(\begin{array}{cc}d & -b \\ -c & a\end{array}\right) \in S L_{2}(Z)$ induces an action

$$
F\left(g_{0}(t, \tau)\right)=F\left(\frac{t}{-c \tau+a}, \frac{d \tau-b}{-c \tau+a}\right) .
$$

Now, if $t=(n / l)(c \tau+d)$ is a polar divisor of $F(t, \tau)$, then one polar divisor of $F\left(g_{0}(t, \tau)\right)$ is given by

$$
\frac{t}{-c \tau+a}=\frac{n}{l}\left(c \frac{d \tau-b}{-c \tau+a}+d\right)
$$

which gives $t=n / l$.

By Lemma 2.7, since $\operatorname{dim} F=1$, we know that up to some constant, $F\left(g_{0}(t, \tau)\right)$ is still one of $F, F^{V}$. This contradicts Lemma 2.8, therefore, this completes the proof of Theorem 2.5.

Remark: We may consider Theorem 2.5 as an odd analogue of rigidity theorems for even dimensional manifolds with isolated fixed points. When $\operatorname{dim} F>1$, by Lemma 2.7, in order to prove rigidity theorems, we need prove Lemma 2.8 for $\Phi_{\tau}^{*} F$ and $\Phi_{\tau}^{*} F^{V}$. That is equivalent to prove Lemma 2.8 for $\Phi_{\tau}^{*} F^{\left[m_{0}\right]}$ and $\left(\Phi_{\tau}^{*} F^{V}\right)^{\left[m_{0}\right]}$. But it is not an equivariant index of some elliptic operator, so Lemma 2.8 does not apply.

\section{JACOBI FORMS AND VANISHING THEOREMS}

In this section, we generalize the rigidity theorems in the previous section to the nonzero anomaly case, from which we derive some holomorphic Jacobi forms. As corollaries, we get many vanishing theorems for odd dimensional spin manifolds, especially an odd $\widehat{U}$-vanishing theorem for loop space. In this section, we will give odd analogues of some results of Section 3 in [Li2].

Recall that the equivariant cohomology group $H_{S^{1}}^{*}(X, \mathbf{Z})$ of $X$ is defined by

$$
H_{S^{1}}^{*}(X, \mathbf{Z})=H^{*}\left(X \times_{S^{1}} E S^{1}, \mathbf{Z}\right),
$$


where $E S^{1}$ is the usual universal $S^{1}$-principle bundle over the classifying space $B S^{1}$ of $S^{1}$. So $H_{S^{1}}^{*}(X, \mathbf{Z})$ is a module over $H^{*}\left(B S^{1}, Z\right)$ induced by projection $\pi: X \times_{S^{1}} E S^{1} \rightarrow B S^{1}$. Similarly an $S^{1}$-bundle $V$ over $X$ extends to bundle $V \times_{S^{1}} E S$ over $X \times_{S^{1}} E S^{1}$, then the equivariant characteristic classes of $V$ are defined by the characteristic classes of $V \times_{S^{1}} E S$ in $H_{S^{1}}^{*}(X, \mathbf{Z})$. Let $u$ be the generator of $H^{*}\left(B S^{1}, Z\right)$. We still use the notation of Section 2. We suppose that there exists some integer $n \in Z$ such that

$$
p_{1}(V)_{S^{1}}-p_{1}(T X)_{S^{1}}=n . \pi^{*} u^{2} .
$$

where we call $n$ the anomaly to rigidity as in [Li 2, §3].

Similar to [Li1], we introduce the following elements in $K(X)\left[\left[q^{\frac{1}{2}}\right]\right]$ :

$$
\begin{gathered}
\Theta_{q}^{\prime}(T X \mid V)_{\nu}=\otimes_{n=1}^{\infty} \wedge_{q^{n}}(V-\operatorname{dim} V) \otimes_{m=1}^{\infty} S_{q^{m}}(T X-\operatorname{dim} X+1), \\
\Theta_{q}(T X \mid V)_{\nu}=\otimes_{n=1}^{\infty} \wedge_{-q^{n-\frac{1}{2}}}(V-\operatorname{dim} V) \otimes_{m=1}^{\infty} S_{q^{m}}(T X-\operatorname{dim} X+1), \\
\Theta_{-q}(T X \mid V)_{\nu}=\otimes_{n=1}^{\infty} \wedge_{q^{n-\frac{1}{2}}}(V-\operatorname{dim} V) \otimes_{m=1}^{\infty} S_{q^{m}}(T X-\operatorname{dim} X+1), \\
\Theta_{q}^{\star}(T X \mid V)_{\nu}=\otimes_{n=1}^{\infty} \wedge_{-q^{n}}(V-\operatorname{dim} V) \otimes_{m=1}^{\infty} S_{q^{m}}(T X-\operatorname{dim} X+1) .
\end{gathered}
$$

For $h=e^{2 \pi i t}, q=e^{2 \pi i \tau}$, with $(t, \tau) \in \mathbf{R} \times \mathbf{H}$, we denote the equivariant index of $T_{g} \otimes \Delta(V) \otimes \Theta_{q}^{\prime}(T X \mid V)_{\nu}, T_{g} \otimes \Theta_{q}(T X \mid V)_{\nu}, T_{g} \otimes \Theta_{-q}(T X \mid V)_{\nu}$ $T_{g} \otimes\left(\Delta^{+}(V)-\Delta^{-}(V)\right) \otimes \Theta_{q}^{\star}(T X \mid V)_{\nu}, T_{g} \otimes \otimes_{m=1}^{\infty} S_{q^{m}}(T X-\operatorname{dim} X+1)$ by $2^{k} F_{d_{s}, \nu}^{V}(t, \tau), F_{D, \nu}^{V}(t, \tau), F_{-D, \nu}^{V}(t, \tau), \quad(-1)^{k} F_{D^{*}, \nu}^{V}(t, \tau), H(t, \tau)$ respectively. We can extend these functions to meromorphic functions on the complex plane. For $\alpha=1,2,3$, let

$$
\theta^{\prime}(0, \tau)=\left.\frac{\partial}{\partial t} \theta(t, \tau)\right|_{t=0}, \theta_{\alpha}(0, \tau)=\left.\theta_{\alpha}(t, \tau)\right|_{t=0} .
$$

Similar to (2.13), we can get

$$
\begin{gathered}
F_{d_{s}, \nu}^{V}(t, \tau)=(2 \pi)^{-r} \frac{\theta^{\prime}(0, \tau)^{r}}{\theta_{1}(0, \tau)^{k}} F_{d_{s}}^{V}(t, \tau) ; \\
F_{D, \nu}^{V}(t, \tau)=(2 \pi)^{-r} \frac{\theta^{\prime}(0, \tau)^{r}}{\theta_{2}(0, \tau)^{k}} F_{D}^{V}(t, \tau) ; \\
F_{-D, \nu}^{V}(t, \tau)=(2 \pi)^{-r} \frac{\theta^{\prime}(0, \tau)^{r}}{\theta_{3}(0, \tau)^{k}} F_{-D}^{V}(t, \tau) ; \\
F_{D^{*}, \nu}^{V}(t, \tau)=(2 \pi)^{k-r} \theta^{\prime}(0, \tau)^{r-k} F_{D^{*}}^{V}(t, \tau) ; \\
H(t, \tau)=c_{1} \operatorname{ch}(g) \prod_{l=1}^{q_{0}} \frac{\pi y_{l}}{\theta\left(y_{l}, \tau\right)} \frac{\theta^{\prime}(0, \tau)^{r}}{\prod_{j=1}^{s} \theta\left(m_{j} t+x_{j}, \tau\right)}[F]
\end{gathered}
$$


where $c_{1}$ is a constant.

Recall that a (meromorphic) Jacobi form of index $m$ and weight $l$ over $L \rtimes \Gamma$, where $L$ is an integral lattice in the complex plane $\mathbf{C}$ preserved by the modular subgroup $\Gamma \subset S L_{2}(\mathbf{Z})$, is a (meromorphic) function $F(t, \tau)$ on $\mathbf{C} \times \mathbf{H}$ such that

$$
\begin{gathered}
F\left(\frac{t}{c \tau+d}, \frac{a \tau+b}{c \tau+d}\right)=(c \tau+d)^{l} e^{2 \pi i m\left(c t^{2} /(c \tau+d)\right)} F(t, \tau), \\
F(t+\lambda \tau+\mu, \tau)=e^{-2 \pi i m\left(\lambda^{2} \tau+2 \lambda t\right)} F(t, \tau),
\end{gathered}
$$

where $(\lambda, \mu) \in L$ and $\left(\begin{array}{ll}a & b \\ c & d\end{array}\right) \in \Gamma$. If $F$ is holomorphic on $\mathbf{C} \times \mathbf{H}$, we say that $F$ is a holomorphic Jacobi form.

Recall the three modular subgroups

$$
\begin{gathered}
\Gamma_{0}(2)=\left\{\left(\begin{array}{ll}
a & b \\
c & d
\end{array}\right) \in S L_{2}(Z) \mid c \equiv 0(\bmod 2)\right\}, \\
\Gamma^{0}(2)=\left\{\left(\begin{array}{ll}
a & b \\
c & d
\end{array}\right) \in S L_{2}(Z) \mid b \equiv 0(\bmod 2)\right\}, \\
\Gamma_{\theta}=\left\{\left(\begin{array}{cc}
a & b \\
c & d
\end{array}\right) \in S L_{2}(Z) \mid\left(\begin{array}{ll}
a & b \\
c & d
\end{array}\right) \equiv\left(\begin{array}{ll}
1 & 0 \\
0 & 1
\end{array}\right) \text { or }\left(\begin{array}{ll}
0 & 1 \\
1 & 0
\end{array}\right)(\bmod 2)\right\} .
\end{gathered}
$$

Now we state the following theorem which is a generalization of Theorem 2.5 to the nonzero anomaly case.

Theorem 3.1. a)For an odd dimensional spin manifold with nontrivial $S^{1}$ action, assume that it has only 1-dimensional fixed points submanifolds and $p_{1}(V)_{S^{1}}$ $p_{1}(T X)_{S^{1}}=n . \pi^{*} u^{2}$ with $n \in \mathbf{Z}$, then $F_{d_{s}, \nu}^{V}(t, \tau), F_{D, \nu}^{V}(t, \tau), F_{-D, \nu}^{V}(t, \tau)$ are holomorphic Jacobi forms of index $\frac{n}{2}$ and weight $r$ over $(2 \mathbf{Z})^{2} \rtimes \Gamma$ with $\Gamma$ equal to $\Gamma_{0}(2), \Gamma^{0}(2), \Gamma_{\theta}$ respectively, and $F_{D^{*}, \nu}^{V}(t, \tau)$ is a holomorphic Jacobi form of index $\frac{n}{2}$ and weight $r-k$ over $(2 \mathbf{Z})^{2} \rtimes S L_{2}(\mathbf{Z})$.

b) If $p_{1}(X)_{S^{1}}=-n u^{2}$, then $H(t, \tau)$ is a holomorphic Jacobi form of index $\frac{n}{2}$ and weight $r$ over $(2 \mathbf{Z})^{2} \rtimes S L_{2}(\mathbf{Z})$.

In this Section, we always assume $\operatorname{dim} F=1$. Similar to Lemma 3.2 in [Li2] and using the same trick in Lemma 2.7, we have: 
Lemma 3.2. a) The following identities hold

$$
\begin{gathered}
F_{d_{s}, \nu}^{V}(t, \tau+1)=F_{d_{s}, \nu}^{V}(t, \tau) ; F_{D, \nu}^{V}(t, \tau+1)=F_{-D, \nu}^{V}(t, \tau) ; \\
F_{D^{*}, \nu}^{V}(t, \tau+1)=F_{D^{*}, \nu}^{V}(t, \tau) ; H(t, \tau+1)=H(t, \tau) .
\end{gathered}
$$

b) If $p_{1}(V)_{S^{1}}-p_{1}(T X)_{S^{1}}=n \cdot \pi^{*} u^{2}$, then

$$
\begin{gathered}
F_{d_{s}, \nu}^{V}\left(\frac{t}{\tau},-\frac{1}{\tau}\right)=\tau^{r} e^{\pi i n t^{2} / \tau} F_{D, \nu}^{V}(t, \tau) ; \\
F_{-D, \nu}^{V}\left(\frac{t}{\tau},-\frac{1}{\tau}\right)=\tau^{r} e^{\pi i n t^{2} / \tau} F_{-D, \nu}^{V}(t, \tau) ; \\
F_{D^{*}, \nu}^{V}\left(\frac{t}{\tau},-\frac{1}{\tau}\right)=\tau^{r} e^{\pi i n t^{2} / \tau} F_{D^{*}, \nu}^{V}(t, \tau) .
\end{gathered}
$$

c) If $p_{1}(X)_{S^{1}}=-n u^{2}$, then

$$
H\left(\frac{t}{\tau},-\frac{1}{\tau}\right)=\tau^{r} e^{\pi i n t^{2} / \tau} H(t, \tau) .
$$

Proof. By (3.5) and (2.29) and the equality $\theta^{\prime}(0, \tau+1)=e^{\frac{\pi i}{4}} \theta^{\prime}(0, \tau)$, we can prove (3.8). Consider the condition on the first equivariant Pontrjagin classes implies the equality

$$
\sum_{\nu=1}^{k}\left(u_{\nu}+n_{\nu} t\right)^{2}-\sum_{l=1}^{q_{0}} y_{l}^{2}-\sum_{j=1}^{s}\left(x_{j}+m_{j} t\right)^{2}=n t^{2},
$$

which gives the equalities

$$
\begin{gathered}
\sum_{\nu=1}^{k} n_{\nu} u_{\nu}=\sum_{j=1}^{s} m_{j} x_{j} \\
\sum_{\nu=1}^{k} u_{\nu}^{2}=\sum_{l=1}^{q_{0}} y_{l}^{2}+\sum_{j=1}^{s} x_{j}^{2} \\
\sum_{\nu=1}^{k} n_{\nu}^{2}-\sum_{j=1}^{s} m_{j}^{2}=n .
\end{gathered}
$$


By (3.5),(2.16),(3.12) and $\operatorname{dim} F=1$, we have

$$
\begin{aligned}
& F_{d_{s}, \nu}^{V}\left(\frac{t}{\tau},-\frac{1}{\tau}\right) \\
= & (2 \pi)^{-r} \frac{\theta^{\prime}\left(0,-\frac{1}{\tau}\right)^{r}}{\theta_{1}\left(0,-\frac{1}{\tau}\right)^{k}} \operatorname{ch}(g) \prod_{l=1}^{q_{0}} \frac{\pi y_{l}}{\theta\left(y_{l},-\frac{1}{\tau}\right)} \frac{\prod_{\nu=1}^{k} \theta_{1}\left(n_{\nu} t / \tau+u_{\nu},-1 / \tau\right)}{\prod_{j=1}^{s} \theta\left(m_{j} t / \tau+x_{j},-1 / \tau\right)}[F] \\
= & (2 \pi)^{-r} \frac{(\tau / i)^{3 r / 2} \theta^{\prime}(0, \tau)^{r}}{(\tau / i)^{k / 2} \theta_{2}(0, \tau)^{k}} \operatorname{ch}(g) \prod_{l=1}^{q_{0}} \frac{\pi y_{l}}{\frac{1}{i} \sqrt{\frac{\tau}{i}} e^{\pi i \tau^{2} y_{l}^{2} / \tau} \theta\left(\tau y_{l}, \tau\right)} \\
& \times \frac{\prod_{\nu=1}^{k} \sqrt{\frac{\tau}{i}} e^{\pi i\left(n_{\nu} t+\tau u_{\nu}\right)^{2} / \tau} \theta_{2}\left(n_{\nu} t+\tau u_{\nu}, \tau\right)}{\prod_{j=1}^{s} \frac{1}{i} \sqrt{\frac{\tau}{i}} e^{\pi i\left(m_{j} t+\tau x_{j}\right)^{2} / \tau} \theta\left(m_{j} t+\tau x_{j}, \tau\right)}[F] \\
= & (2 \pi)^{-r} \tau^{r} e^{\pi i n t^{2} / \tau} \frac{\theta^{\prime}(0, \tau)^{r}}{\theta_{2}(0, \tau)^{k}} \operatorname{ch}(g)^{[1]}\left[\prod_{l=1}^{q_{0}} \frac{\pi y_{l}}{\theta\left(\tau y_{l}, \tau\right)} \frac{\prod_{\nu=1}^{k} \theta_{2}\left(n_{\nu} t+\tau u_{\nu}, \tau\right)}{\prod_{j=1}^{s} \theta\left(m_{j} t+\tau x_{j}, \tau\right)}\right]^{\left[2 q_{0}\right]}[F] \\
= & (2 \pi)^{-r} \tau^{r} e^{\pi i n t^{2} / \tau} \frac{\theta^{\prime}(0, \tau)^{r}}{\theta_{2}(0, \tau)^{k}} \operatorname{ch}(g)^{[1]}\left[\prod_{l=1}^{q_{0}} \frac{\pi y_{l}}{\theta\left(y_{l}, \tau\right)} \frac{\prod_{\nu=1}^{k} \theta_{2}\left(n_{\nu} t+u_{\nu}, \tau\right)}{\prod_{j=1}^{s} \theta\left(m_{j} t+x_{j}, \tau\right)}\right]^{\left[2 q_{0}\right]}[F] \\
= & \tau^{r} e^{\pi i n t^{2} / \tau} F_{D, \nu}^{V}(t, \tau) ;(3.13)
\end{aligned}
$$

Using the same trick, we can prove the other identities.

Lemma 3.3 a)For an odd dimensional spin manifold with nontrivial $S^{1}$ action, assume that it has only 1-dimensional fixed points submanifolds and $p_{1}(V)_{S^{1}}-$ $p_{1}(T X)_{S^{1}}=n . \pi^{*} u^{2}$ with $n \in \mathbf{Z}$, then $F_{d_{s}, \nu}^{V}(t, \tau), F_{D, \nu}^{V}(t, \tau), F_{-D, \nu}^{V}(t, \tau)$ are Jacobi forms of index $\frac{n}{2}$ and weight $r$ over $(2 \mathbf{Z})^{2} \rtimes \Gamma$ with $\Gamma$ equal to $\Gamma_{0}(2), \Gamma^{0}(2), \Gamma_{\theta}$ respectively, and $F_{D^{*}, \nu}^{V}(t, \tau)$ is a holomorphic Jacobi form of index $\frac{n}{2}$ and weight $r-k$ over $(2 \mathbf{Z})^{2} \rtimes S L_{2}(\mathbf{Z})$.

b) If $p_{1}(X)_{S^{1}}=-n u^{2}$, then $H(t, \tau)$ is a Jacobi form of index $\frac{n}{2}$ and weight $r$ over $(2 \mathbf{Z})^{2} \rtimes S L_{2}(\mathbf{Z})$.

Proof: Using (2.19),(3.5) and (3.12), similar to Lemma 2.6 b), we can prove these $F^{V}$ and $H$ satisfy the second equation of the definition of Jacobi form (3.6).

Recall that $T$ and $S T^{2} S T$ generate $\Gamma_{0}(2)$, and also $\Gamma^{0}(2)$ and $\Gamma_{\theta}$ are conjugate to $\Gamma_{0}(2)$ by $S$ and $S T$, respectively. Then by Lemma 3.2, we can get that these $F_{\nu}^{V}$ and $H$ satisfy the first equation of the definition of Jacobi form (3.6). 


$$
\begin{aligned}
& \text { For } g_{0}=\left(\begin{array}{cc}
a & b \\
c & d
\end{array}\right) \in S L_{2}(Z) \text {, we write } \\
& \left.F\left(g_{0}(t, \tau)\right)\right|_{m, l}=(c \tau+d)^{-l} e^{-2 \pi i m c t^{2} /(c \tau+d)} F\left(\frac{t}{c \tau+d}, \frac{a \tau+b}{c \tau+d}\right),
\end{aligned}
$$

which denotes the action of $g_{0}$ on a Jacobi form $F$ of index $m$ and weight $l$.

By Lemma 3.2, for $F \in\left\{F_{\nu}^{V}, H\right\}$, its modular transformation $\left.F\left(g_{0}(t, \tau)\right)\right|_{\frac{n}{2}, r}$ (or $\left.F\left(g_{0}(t, \tau)\right)\right|_{\frac{n}{2}, r-k}$ ) is still one of the $F \in\left\{F_{\nu}^{V}, H\right\}$. Similar to Lemma 2.8, we have the following Lemma

Lemma 3.4 For any $g_{0} \in S L_{2}(Z)$, let $F$ be one of the $\left\{F_{\nu}^{V}\right\}$ or $H$, then $\left.F\left(g_{0}(t, \tau)\right)\right|_{\frac{n}{2}, r}\left(\right.$ or $\left.\left.F\left(g_{0}(t, \tau)\right)\right|_{\frac{n}{2}, r-k}\right)$ is holomorphic on $\mathbf{R} \times \mathbf{H}$.

The proof of Theorem 3.1: By (3.14), $\left.F\left(g_{0}(t, \tau)\right)\right|_{m, l}$ and $F\left(\frac{t}{c \tau+d}, \frac{a \tau+b}{c \tau+d}\right)$ have the same poles. As in [Li2] or [LiM], by Lemma 3,4, similar to the proof of Theorem 2.5, we may prove Theorem 3.1.

Next we will prove a vanishing theorem for loop space. The following lemma is established in [EZ, Theorem 1.2].

Lemma 3.5 Let $F$ be a holomorphic Jacobi form of index $m$ and weight $l$. Then for fixed $\tau, F(t, \tau)$, if not identically zero, has exactly $2 m$ zeros in any fundamental domain for the action of the lattice on $\mathbf{C}$. when $m=1$ and $l$ is odd, then $F$ is identically zero

By this lemma, if $m<0$, then $F$ must be identically zero. If $m=0$, then $F$ must be independent of $t$.

Corollary 3.6 Let $X, V, n$ and the assumption condition be as in theorem 3.1. If $n=0$, the equivariant index of the Toeplitz operators in Theorem 3.1 are independent of $h \in S^{1}$. If $n<0$ or $n=2$ and $r=\frac{\operatorname{dim} X-1}{2}$ is odd, then these equivariant index are identically zero; in particular, the index of these Toeplitz operators is zero. 
The following theorem is an odd analogue of $\widehat{U}$-vanishing theorem for loop space.

Theorem 3.7 Let $M$ be a compact odd dimensional spin manifold with a nontrivial $S^{1}$ action and 1-dimensional fixed points submanifolds. If $p_{1}(X)_{S^{1}}=-n \pi^{*} u^{2}$, then the Lefschetz number, especially the index of $T_{g} \otimes \otimes_{m=1}^{\infty} S_{q^{m}}(T X-\operatorname{dim} X+1)$ is zero.

Proof: By $p_{1}(X)_{S^{1}}=-n \pi^{*} u^{2}$, then $\sum m_{j}^{2}=-n$, thus $n \leq 0$. When $n=0$, then $m_{j}=0$ and $S^{1}$-action has no fixed point. By $(3.5), H(t, \tau)$ is zero. When $n<0$, by Theorem $3.1 \mathrm{~b}$ ) and Lemma $3.5, H(t, \tau)$ is also zero.

\section{An EQUiVARIANT ODD INDEX THEOREM FOR DiRAC OPERATORS With INVOLUTION PARITY}

In this section, we will prove an equivariant odd index theorem which is the generalization of Freed's odd index theorem in [Fr] by a direct geometric method in [LYZ] ( also see [Wa]). As in Section 2, Let $X$ be a closed (connected for simplicity) oriented spin manifold of dimension $2 r+1$, with a fixed spin structure and $\triangle(T X)$ be the canonical complex spinor bundle of $X$. A compact group $\widetilde{H}$ of isometries of $X$ preserves the orientation and spin structure. Let $E$ be a $\widetilde{H}$-vector bundle with $\widetilde{H}$-invariant Hermitian connection $\nabla^{E}$. There is a lift of $h \in \widetilde{H}$ acting on $\Gamma(\triangle(T X) \otimes E)$ which commutes with $D^{E}$.

Suppose that $\widehat{\tau}: X \rightarrow X$ is an orientation-reversing isometric involution which preserves the Pin structure induced by the Spin structure and commutes with any $h \in \widetilde{H}$. We also assume that there is a lift of $\widehat{\tau}$ on $E$ which commutes with any $h \in \widetilde{H}$. We may take $\nabla^{E}$ is also $\widehat{\tau}$-invariant. As in $[\mathrm{Fr}]$ and [Wa], we have there exists a self-adjoint lift $\widehat{\tau}: \Gamma(X ; \Delta(T X) \otimes E) \rightarrow \Gamma(X ; \Delta(T X) \otimes E)$ satisfying

$$
\widehat{\tau}^{2}=1 ; D^{E} \widehat{\tau}=-\widehat{\tau} D^{E}, \widehat{\tau} h=h \widehat{\tau}
$$

Then the +1 and -1 eigenspaces of $\widehat{\tau}$ give a splitting of the twisted spinor fields

$$
\Gamma(X ; \Delta(T X) \otimes E) \cong \Gamma^{+}(X ; \Delta(T X) \otimes E) \bigoplus \Gamma^{-}(X ; \Delta(T X) \otimes E)
$$

and the Dirac operator interchanges $\Gamma^{+}(X ; \Delta(T X) \otimes E)$ and $\Gamma^{-}(X ; \Delta(T X) \otimes$ $E)$. By (4.1), $h$ preserves $\Gamma_{-}^{+}(X ; \Delta(T X) \otimes E)$. The purpose of this section is to compute the equivariant index

$$
\operatorname{index}_{h}\left[D^{E,+}: \Gamma^{+}(X ; \Delta(T X) \otimes E) \rightarrow \Gamma^{-}(X ; \Delta(T X) \otimes E)\right]
$$


The simplest example is $X=M \times S^{1}$ with $\tau$ the reflection $x \rightarrow-x$ on $S^{1}$ and $\widetilde{H}=S^{1}$ acting on the spin manifold $M$. By the equivariant index theorem in the following (cf. Theorem 4.1), we have $\operatorname{Ind}_{h} D^{+}=\operatorname{Ind}_{h} D^{M}$.

By the Mckean-Singer formula, we have

$$
\operatorname{Ind}_{h}\left(D^{E,+}\right)=\operatorname{Tr}\left(\widehat{\tau} h e^{-t\left(D^{E}\right)^{2}}\right)=\operatorname{Tr}\left(e^{-t\left(D^{E}\right)^{2}} h \widehat{\tau}\right)
$$

Let $T=h \widehat{\tau}$ be an orientation-reversing isometric, then $T$ has a lift $\widehat{T}$ on $\Gamma^{+}(X ; \Delta(T X) \otimes$ $E$ ). Next, for $T$ and the twisted coefficient $E$, using the method in [LYZ] (also see [Wa]), we can get the following index formula. For simplicity, we also assume that $T$ has only one fixed point component $F_{0}$ with dimension $2 q_{0}$ and codimension $2 s_{0}+1$

Theorem 4.1. We have

$$
\operatorname{Ind}_{h}\left(D^{\widehat{\tau}} \otimes E\right)=\left[c_{0} \widehat{A}\left(F_{0}\right)\left[\operatorname{Pf}\left(2 \sin \left(\frac{R^{N\left(F_{0}\right)}}{4 \pi \sqrt{-1}}+\frac{1}{2} \Theta\right)\right)\right]^{-1} \operatorname{ch}_{T}(E)\right]\left[F_{0}\right],
$$

where $c_{0}$ is a constant and $\Theta$ is the rotation matrix of $T$. Here the Pfaffian is taken on the first $2 s_{0}$ coordinates of $N\left(F_{0}\right)$.

When $h=$ id, we get the Freed odd index theorem. By the Lichnerowicz formula for Dirac operators, we get

Corollary 4.2. Let $X$ be an odd dimensional compact oriented Spin manifold and $\widehat{\tau}: X \rightarrow X$ be an orientation-reversing isometric involution which preserves Pin structure. Suppose that $F_{1}, \cdots, F_{\widehat{r}}$ are components of the fixed point set with codimension $2 m_{j}^{0}+1$ and $\operatorname{ch} \Delta\left(N_{j}\right)$ is the Chern character of the spinor bundle on $N\left(F_{j}\right)$. If the scalar curvature of $X$ is nonnegative and positive at one point then

$$
\sum_{j=1}^{\widehat{r}} \int_{F_{j}}(\sqrt{-1})^{m_{1}^{0}-m_{j}^{0}} \widehat{A}\left(T F_{j}\right)\left[\operatorname{ch} \triangle\left(N_{j}\right)\right]^{-1}=0 .
$$

Now we let $\widetilde{H}=S^{1}$ and $h=e^{2 \pi i t}$ is a generator of $S^{1}$, then we have

Lemma 4.3. The following statements hold: 
a) Let $F(\cdot)$ denote a fix point set, then

$$
F\left(h^{m} \widehat{\tau}\right)=F(h \widehat{\tau})=F\left(S^{1}\right) \cap F(\widehat{\tau}), \text { for any } m \in Z,
$$

b) The normal bundle $N(\widehat{\tau} h)$ has a $\widehat{\tau} S^{1}$-invariant decomposition $N(\widehat{\tau} h)=N_{0} \oplus_{1 \leq j}$ $N_{j}$ where $N_{0}$ is a real vector bundle and $N_{j}$ is an oriented 2-plane bundle and $\widehat{\tau} h$ acts on $N_{0}$ by -1 and $N_{j}$ by $e^{2 \pi i\left(m_{j} t+a_{j}\right)}$, where $m_{j} \neq 0 \in Z$ and $a_{j}=0$ or $\frac{1}{2}$.

Proof. a) If $h \widehat{\tau} x=x$, then $(h \widehat{\tau})^{2} x=x$. Since $\widehat{\tau}^{2}=$ id; $\widehat{\tau} h=h \widehat{\tau}$, we know $h^{2} x=x$. Since $F\left(h^{m}\right)=F(h)=F\left(S^{1}\right)$, we have $h x=x$ and $\widehat{\tau} x=x$. Thus $F(h \widehat{\tau}) \subset F\left(S^{1}\right) \cap F(\widehat{\tau})$ and the inverse inclusion is trivial. Similarly we can get $F\left(h^{m} \widehat{\tau}\right)=F\left(S^{1}\right) \cap F(\widehat{\tau})$.

b) By a), we have $N(h \widehat{\tau})$ is a $S^{1}$-representative bundle, so $N(h \widehat{\tau})$ has a $S^{1}$ invariant decomposition $N_{0} \oplus_{1 \leq j} N_{j}$ and $N_{0}$ is a real vector bundle and $N_{j}$ is an oriented 2-plane bundle and $h$ acts on $N_{0}$ by 1 and $N_{j}$ by $e^{2 \pi i m_{j} t}$, where $m_{j} \neq 0 \in Z$. Since $\widehat{\tau}$ is an involution isometry and commutes with $h$, so $\widehat{\tau}$ preserves the above decomposition acting by +1 or -1 .

Now we give a Chern root expression of (4.5) for $\widetilde{H}=S^{1}$ case. We assume $E$ has also $h \widehat{\tau}$-invariant decomposition $\oplus E_{\nu}$ and $h \widehat{\tau}$ acts on $E_{\nu}$ by $e^{2 \pi i\left(c_{\nu} t+b_{\mu}\right)}$, where $c_{\nu} \in \mathbf{Z}$ and $b_{\mu}=0$ or $\frac{1}{2}$. Let $2 \pi z_{\nu}$ is the Chern root of $E_{\nu}$, then we have:

$\operatorname{Ind}_{h}\left(D^{\widehat{\tau}} \otimes E\right)=\left[c_{0} \prod_{l=1}^{q_{0}} \frac{\pi y_{l}}{\sin \pi y_{l}} \prod_{j=1}^{s} \frac{1}{\sin \left[\pi\left(x_{j}+m_{j} t+a_{j}\right)\right]} \sum_{\nu=1}^{l_{0}} e^{2 \pi \sqrt{-1}\left(c_{\nu} t+b_{\mu}+z_{\nu}\right)}\right][F]$.

By Lemma 4.3, similar to the proof of Theorem 2.4, we can get another AtiyahHirzebruch type theorem.

Theorem 4.4. Let $X$ be odd dimensional connected spin manifold which admits an nontrivial $S^{1}$ action and $\widehat{\tau}: X \rightarrow X$ be an orientation-reversing isometric involution which preserves the Pin structure and commutes with $S^{1}$ action, then

$$
\sum_{j=1}^{\widehat{r}} \int_{F_{j}}(\sqrt{-1})^{m_{1}^{0}-m_{j}^{0}} \widehat{A}\left(T F_{j}\right)\left[\operatorname{ch} \triangle\left(N_{j}\right)\right]^{-1}=0 .
$$


Proof. Let $h$ be the topological generator of $S^{1}$. By Lemma 4.3, $F\left(h^{m} \widehat{\tau}\right)=$ $F(h \widehat{\tau})=F\left(S^{1}\right) \cap F(\widehat{\tau})$. By $(4.1),(4.2)$ and $(4.3)$, then $\operatorname{ind}_{S^{1}}\left(D^{\widehat{\tau}}\right):=\left[\operatorname{ker} D^{\widehat{\tau}}\right]-$ $\left[\operatorname{coker} D^{\widehat{\tau}}\right]$ is a virtual representative space of $S^{1}$, then its character

$$
\operatorname{ind}_{z_{0}}\left(D^{\widehat{\tau}}\right)=\sum_{k=-N}^{N} n_{k} z_{0}^{k}
$$

where $z_{0} \in S^{1}$ and $k, n_{k}$ are integers. By (4.7) for untwisted case, for any $z=h^{m} \in \Gamma$, we have up to a constant

$\operatorname{ind}_{z}\left(D^{\widehat{\tau}}\right)=\int_{F\left(S^{1}\right) \cap F(\widehat{\tau})} \widehat{A}\left(F\left(S^{1}\right) \cap F(\widehat{\tau})\right) \sum_{j=1}^{s} \frac{1}{z^{m_{j} / 2} e^{i \pi x_{j}} e^{i \pi a_{j}}-z^{-m_{j} / 2} e^{-i \pi x_{j}} e^{-i \pi a_{j}}}$.

Considering the Taylor expansion of $\frac{1}{z^{m_{j} / 2} e^{a} e^{x}-z^{-m_{j} / 2} e^{-a} e^{-x}}$, we see that the right hand of (4.9) equals

$$
\sum_{j=1}^{s} \frac{1}{z^{m_{j} / 2} e^{\pi i a_{j}}-z^{-m_{j} / 2} e^{-\pi i a_{j}}} \times f\left(\frac{z^{m_{j} / 2} e^{\pi i a_{j}}+z^{-m_{j} / 2} e^{-\pi i a_{j}}}{z^{m_{j} / 2} e^{\pi i a_{j}}-z^{-m_{j} / 2} e^{-\pi i a_{j}}}\right),
$$

where $f\left(\frac{z^{m_{j} / 2} e^{\pi i a_{j}}+z^{-m_{j} / 2} e^{-\pi i a_{j}}}{z^{m_{j} / 2} e^{\pi i a_{j}}-z^{-m_{j} / 2} e^{-\pi i a_{j}}}\right)$ is a polynomial on $\frac{z^{m_{j} / 2} e^{\pi i a_{j}}+z^{-m_{j} / 2} e^{-\pi i a_{j}}}{z^{m_{j} / 2} e^{\pi i a_{j}}-z^{-m_{j} / 2} e^{-\pi i a_{j}}}$. Note that (4.11) also has poles at $|z|=1$, so similar to Theorem 2.4, we can prove Theorem 4.4

Acknowledgement. The work of the first author was partially supported by NSF and NSFC. The work of the second author was supported by NSFC No. 10801027.

\section{References}

[AH] M. F. Atiyah and F. Hirzebruch, Spin manifolds and group actions, in Collected Works, M. F. Atiyah, Vol 3, pp. 417-429.

[BT] R. Bott and C. Taubes, On the rigidity theorems of Witten, J. AMS.(2)(1989),137186.

[Ch] K. Chandrasekharan, Elliptic functions, Grundlehren Math. Wiss.281, Springer, Berlin, 1985.

[EZ] M. Eichler and D. Zagier, The theory of Jacobi forms, Progr. Math. 55. Birkhäuser, Boston, 1985. 
[Fa] H. Fang, Equivariant spectral flow and a Lefschetz theorem on odd-dimensional spin manifolds, Pacific J. Math. 220(2005), 299-312.

[Fr] D. Freed, Two index theorems in odd dimensions, Commu. Anal. Geom. 6(1998), 317-329.

[LYZ] J. D. Lafferty, Y. L. Yu and W. P. Zhang, A direct geometric proof of Lefschetz fixed point formulas, Trans. AMS. 329(1992), 571-583.

[LaM] B. Lawson and M. L. Michelson, Spin geometry, Princeton Univ. Press, 1993.

[Li1]K. Liu, On elliptic genera and theta functions, Topology 35(1996), 617-640.

[Li2] K. Liu, On modular invariance and rigidity theorems, J. Diff. Geom. 41 (1995), 343-396.

[LiM] K. Liu and X. Ma, On family rigidity theorem I, Duke Math. Jour. , 102(2000), 451-473.

[Wa] Y. Wang, Chern-Connes character for the invariant Dirac operator in odd dimensions, Sci. in China Ser. A 48(2005) 1124-1134.

Kefeng Liu

Center of Mathematical Sciences, Zhejiang University Hangzhou Zhejiang 310027, China and Department of Mathematics, University of California at Los Angeles, Los Angeles CA 90095-1555, USA

E-mail: liu@ucla.edu.cn; liu@cms.zju.edu.cn

Yong Wang

School of Mathematics and Statistics, Northeast Normal University, Changchun Jilin, 130024 and Center of Mathematical Sciences, Zhejiang University, Hangzhou Zhejiang 310027, China

E-mail: wangy581@126.com; wangy581@nenu.edu.cn 